Journal of Research in Interprofessional

Practice and

Education

Vol. 3.3

January, 2014
Journal of Research in Interprofessional Practice and Education (JRIPE)

Vol. 3.3

(c) 2014

Corresponding Author: Sophia Gocan, Email: isqocan@tohon.on.cai

\section{Interprofessional Collaboration in Ontario's Family Health Teams: A Review of the Literature}

\author{
Sophia Gocan, RN, MScN; Mary Ann Laplante, RN, BScN; \\ \& A. Kirsten Woodend, RN, BScN, PhD
}

\begin{abstract}
Background: In Ontario, 200 interprofessional Family Health Teams (FHTs) have been established since 2005 to improve primary healthcare access, patient outcomes, and costs. High levels of interprofessional collaboration are important for team success; however, effective team functioning is difficult to achieve. FHTs are in their infancy, and little is known about the determinants that have influenced the quality of team collaboration or the outcomes that FHTs have achieved. The objective of this article is to examine current knowledge regarding FHT team functioning.

Methods and Findings: A search of the literature resulted in eleven articles for final analysis, which were primarily qualitative in nature. A narrative synthesis of study findings was completed. A number of common challenges to interprofessional collaboration were identified. Nevertheless, patients and providers described improved healthcare access, greater satisfaction, and enhanced quality of healthcare using a FHT approach. Collaboration was fostered by effective leadership, communication, outcome evaluation, and training for both professionals and patients alike.

Conclusions: Ontario FHTs have generated improvements in healthcare access and outcomes. Collaborative team functioning, while present, has not reached its full potential. Supportive public policy, education for patients and providers, and evaluation research is needed to advance FHT functioning.

Keywords: Primary healthcare; Interprofessional relations; Family health team
\end{abstract}

\section{Introduction}

The primary care setting serves as a critical entry point to the healthcare system [1]. It acts as a gateway for acute treatments and an essential site for the management of chronic conditions, as well as preventive care, health promotion, and population health initiatives [2]. Across the globe there has been considerable reform, with an increased emphasis on delivering care using a team-based approach [3]. Interprofessional primary care teams include physicians, nurses, pharmacists, and health educators among many other health professionals working side by side in clinical practice [4]. "Collaborative practice is an inter-professional process for communication and decision making that enables the separate and shared knowledge and skills of care providers to synergistically influence the client/patient care provided" [5]. Interprofessional teams improve healthcare access, resource utilization, efficiency of services, outcomes, and costs [6,7]. Patients cared for in interprofessional settings express more satisfaction, as well as enhanced health knowledge, skills, and self-care strategies [6]. In primary care settings, a team approach has been shown to be successful in the prevention and management of mental health conditions and chronic diseases, and has contributed to improvements in health status and quality of life [6]. 
2

Interprofessional Collaboration in Ontario's Family Health Teams: A Review of the Literature

Gocan, Laplante, \& Woodend

Journal of Research in Interprofessional Practice and Education

Vol. 3.3

January, 2014
Until more recently, family physicians alone have delivered the majority of primary healthcare in Canada [8]. A goal was established at the First Minister's Health Accord in 2004 to provide 50\% of Canadians with 24/7 access to primary healthcare, delivered by a multidisciplinary team, by 2011 [1]. With only $10 \%$ of Canadian family physicians working in multidisciplinary practices in 2002 [9], considerable efforts were needed to achieve this target. New models were therefore designed to replace solo primary care practices. These new models offered shared work environments for family physicians with an opportunity for information exchange and collegial support [10]. They also offered patients enhanced access, safety, and quality of healthcare [10].

In practical terms, transitioning to interprofessional team models of care has been challenging. A shift from solo-practitioner to team-based services involves more than merely pulling together a healthcare practice in which multiple disciplines are provided with a mandate to work together. High levels of team functioning are difficult to achieve [11,12]. Effective interprofessional collaboration incorporates several key attributes, including: the engagement of two or more professionals from different disciplines who share a common goal, shared knowledge, multiple interactions over time, an understanding of each professional's role, interdependence among professionals, symmetrical power, and a supportive organizational environment [13].

Almost three decades ago, the province of Quebec established interprofessional Community Health Care Centres (CHCCs), the first of their kind in Canada [12]. Despite their long history, however, a study exploring team functioning at more than 150 CHCCs demonstrated that only moderate levels of interprofessional collaboration have been achieved in these primary care settings [12]. Barriers to interprofessional collaboration include the divided education of health professionals according to discipline, which creates a silo mentality negating the value of professional plurality and limiting the development of mutual understanding and respect [14]. In addition, team vision, hierarchy, professional culture, medico-legal responsibilities, funding and remuneration models, communication systems, clarity of roles, understanding regarding scope of practice, and population health needs have been identified as important factors that influence the intensity of collaborative care $[6,14,15]$.

In the province of Ontario, 200 interprofessional Family Health Teams (FHTs) have been established since 2005 as a transformational strategy to improve the accessibility, effectiveness, and quality of primary care health services across the province [16]. The FHT links physicians with interdisciplinary health professionals (IHPs) such as nurses, dietitians, and pharmacists, among others, to share patient care responsibilities and enhance the delivery of comprehensive, co-ordinated health services [17]. Although physicians are also IHPs, they are described separately in the literature, and as such, this convention has been followed within this article. The aim of FHTs is to enhance healthcare access within their communities and to reduce the strain on emergency departments, providing care for orphan patients (those previously without a family physician) and expanding hours of availability to patients [17]. Goals include the provision of patient-centred care, access to a variety of health professionals, assistance with health system navigation, expanded 
3

Interprofessional Collaboration in Ontario's Family Health Teams: A Review of the Literature

Gocan, Laplante, \& Woodend

Journal of Research in Interprofessional Practice and Education

Vol. 3.3

January, 2014 preventive care services, and chronic care management [17]. FHTs are customized to meet the unique needs of their community. As such, they vary in size, spatial organization, team composition, governance models, partnerships, and range of programs offered. FHTs in Ontario provide healthcare services to approximately 2.7 million people, of which 578,000 previously did not have a regular family physician [16]. These teams are in their infancy, and little is known about the characteristics that have influenced the quality of team collaboration in FHTs or the outcomes Ontario FHTs have achieved using a team-based approach.

It is not known whether Ontario FHTs have been successful in transforming into effective team environments, overcoming common challenges to collaborative team functioning. It is also not known whether there are unique determinants that have particular importance for interprofessional collaboration in this setting. A deeper understanding of the factors that support collaborative interprofessional practice provides an opportunity to explore current FHT functioning, effective strategies for collaborative care, and to identify gaps that need further attention as these models of care progress.

\section{Research objective}

The objective of this article is to report on a review of the literature and examine what is currently known about FHT team functioning in Ontario. Aims include the identification of determinants that have been found to influence the success of interprofessional collaboration and effective team practices within Ontario's FHTs, as well as an assessment of patient, provider, and healthcare system outcomes achieved within FHTs.

\section{Methods}

A literature search was conducted using the MeSH heading "Primary Health Care" and the keyword search term "Family Health Team" within Medline and CINAHL. The search was restricted to the English language and publications after 2005, as this year coincided with the inception of FHTs in Ontario. This search was completed April 10, 2012. It also included review of grey literature, such as thesis and dissertation abstracts, Google Scholar, as well as Canadian and Ontario government and research websites. Bibliographic references from retrieved articles were also hand searched for relevant publications. The abstracts of all articles were screened for inclusion in this analysis by the lead researcher (SG). Articles were included if their focus was on Ontario FHTs and they examined either collaborative team characteristics or FHT outcomes. Articles were excluded for the following reasons: duplication, article based on debate or commentary, study not pertaining to an Ontario FHT, no evaluation of FHT team characteristics or outcomes. Full-text articles retrieved were reviewed according to the same criteria.

The analysis of selected research articles commenced with an exploration of study characteristics, methodology, and outcomes. This was followed by an examination and synthesis of the determinants of interprofessional collaboration and FHT outcomes according to the identified research objectives. No framework for the analysis was used a priori to allow new concepts to be identified. 
4

Interprofessional Collaboration in Ontario's Family Health Teams: A Review of the Literature

Gocan, Laplante, \& Woodend

Journal of Research in Interprofessional Practice and Education

Vol. 3.3

January, 2014

\section{Findings}

Ninety-five articles were identified for review (Figure 1). Four additional articles were generated from a review of the bibliographic references of retrieved articles, Google scholar, and a search of government websites for research reports. No further studies were identified by hand searches of the two journals from which most of the eligible studies were identified (Canadian Family Physician and Journal of Interprofessional Care). After initial screening of titles and abstracts, 38 articles were identified for full-text review. Eleven articles were included in the final analysis.

\section{Figure 1}

\section{Literature Search}

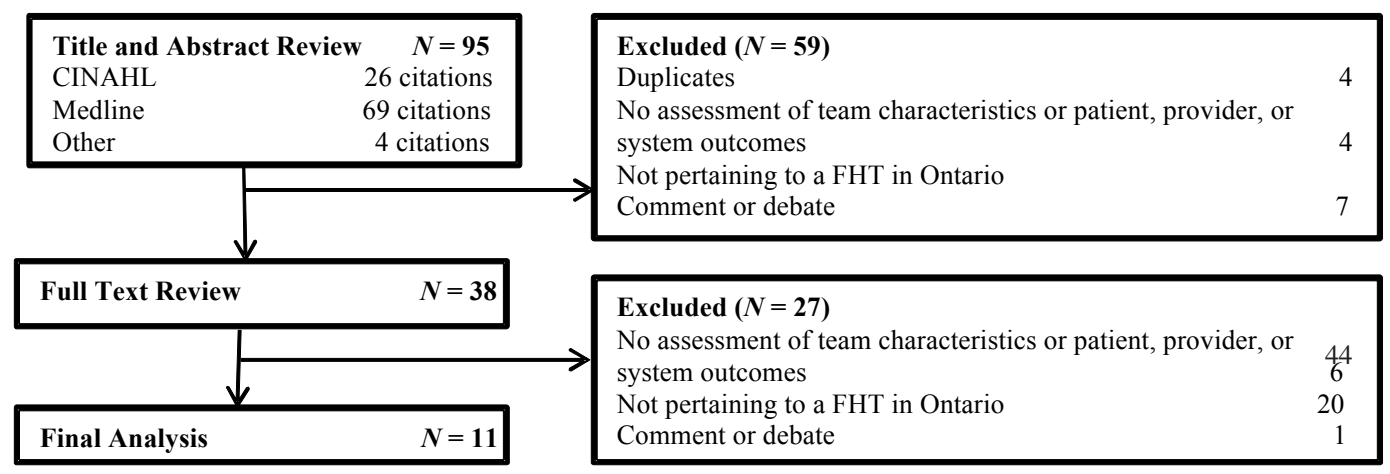

\section{Study characteristics, methodology, and outcomes}

A review of articles included in this analysis is provided in Table 1. Collectively, these 11 studies, published between 2008 and 2011, included 87 FHT organizations, 694 FHT staff members, and 80 patients/family members. All studies except two were qualitative in nature, with the majority using a case study methodology. Two utilized a mixed methods approach. Data collection methods included interviews, surveys, focus groups, observation, chart audits, and documentation review. Eight studies were published in peer-reviewed journals; a further three were obtained from grey literature, including final reports from academic or health research institutions. Among these, eight studies were designed to examine teamwork or interprofessional collaboration. The remaining three studies were focused on patient and physician experiences in transitioning to a FHT setting, the status of integrated mental health services in Northern FHTs, and the impact of integrated memory clinic services in primary care teams.

\section{Important determinants for interprofessional collaboration and effective team relationships in a FHT}

Characteristics identified as important for collaborative team practices were assessed in all 11 studies. Several determinants were identified as important for interprofessional collaboration and effective FHT relationships (Table 2). Factors influencing collaborative team practice stemmed from three different levels of influ- 


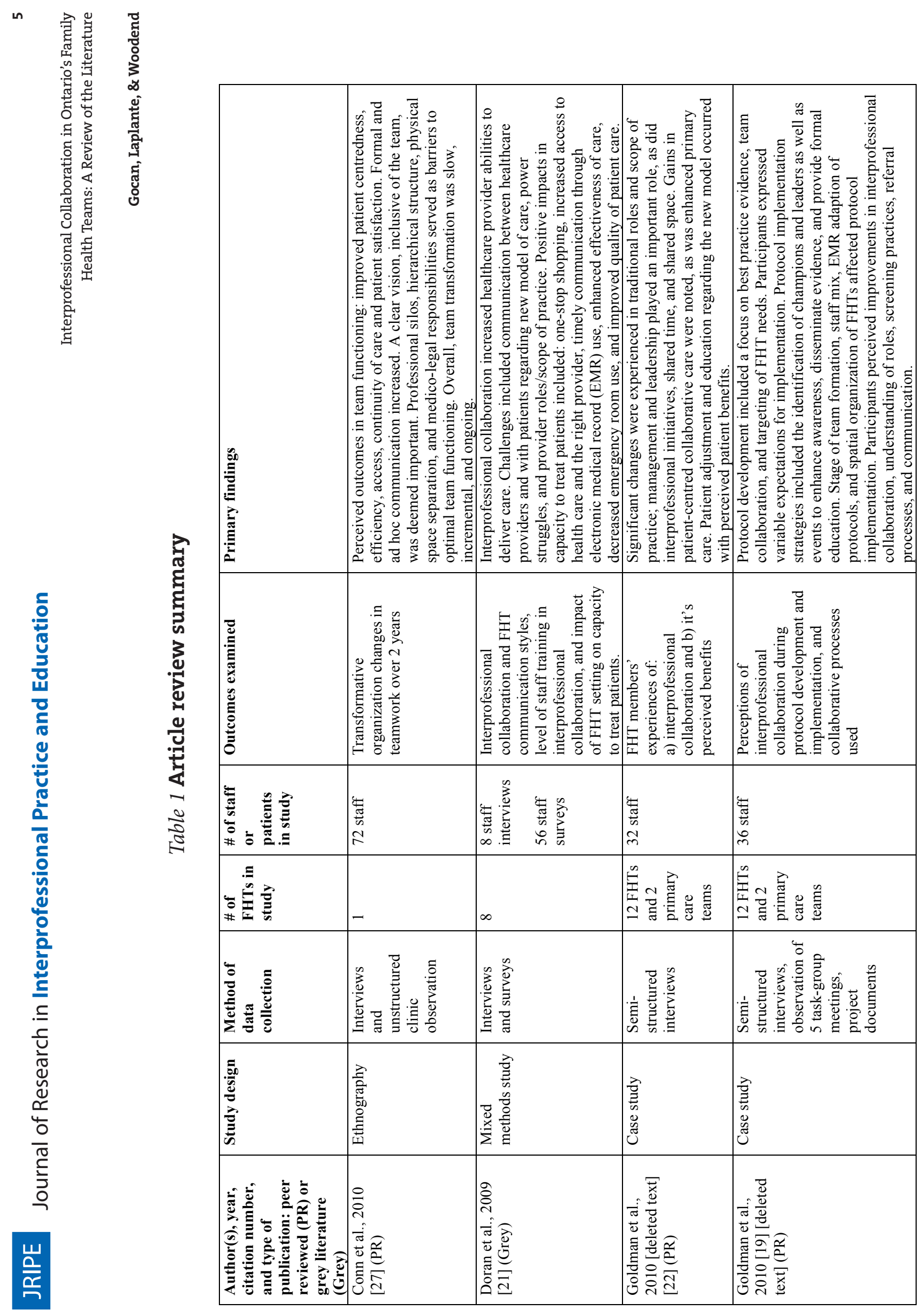

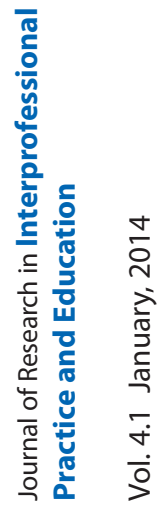




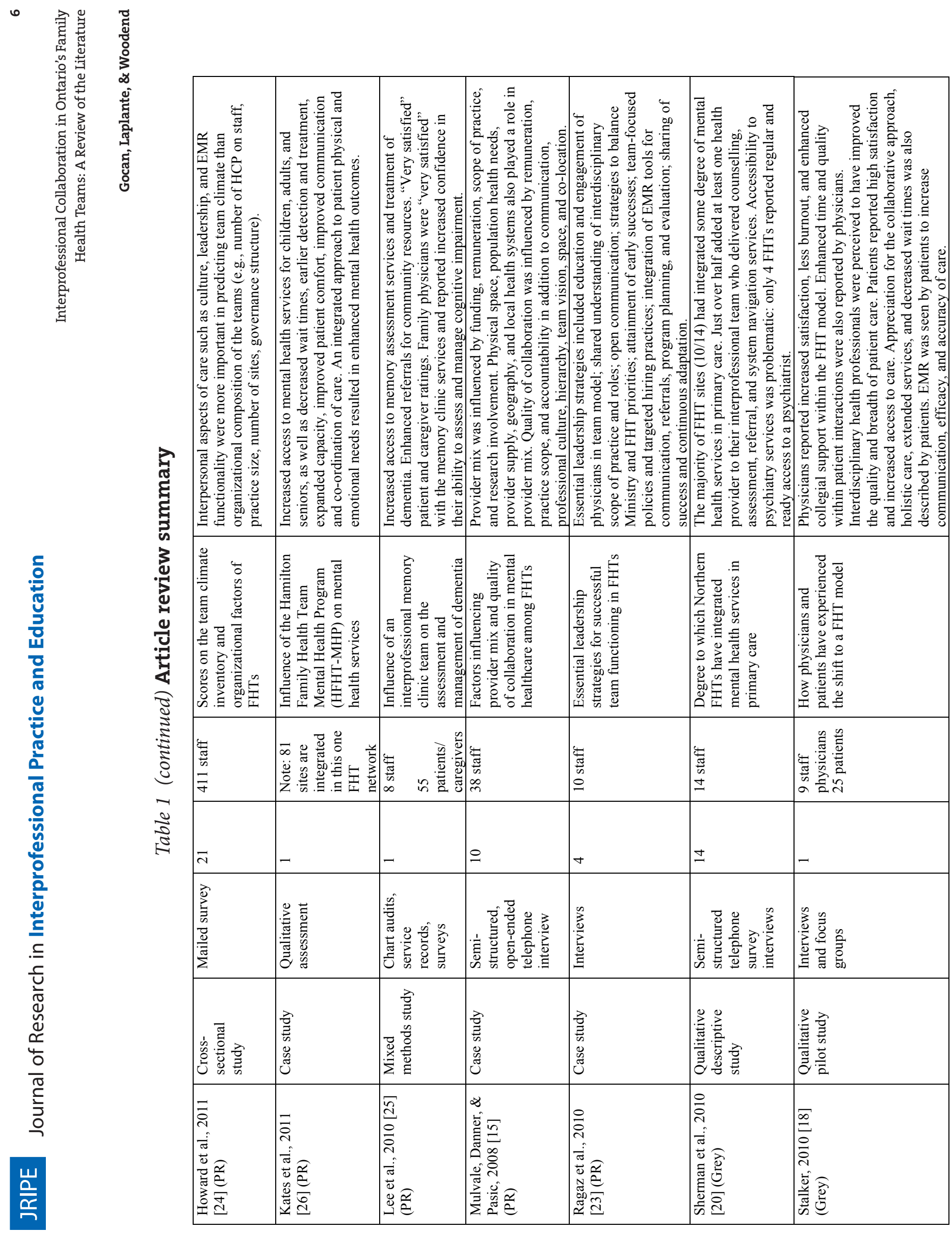




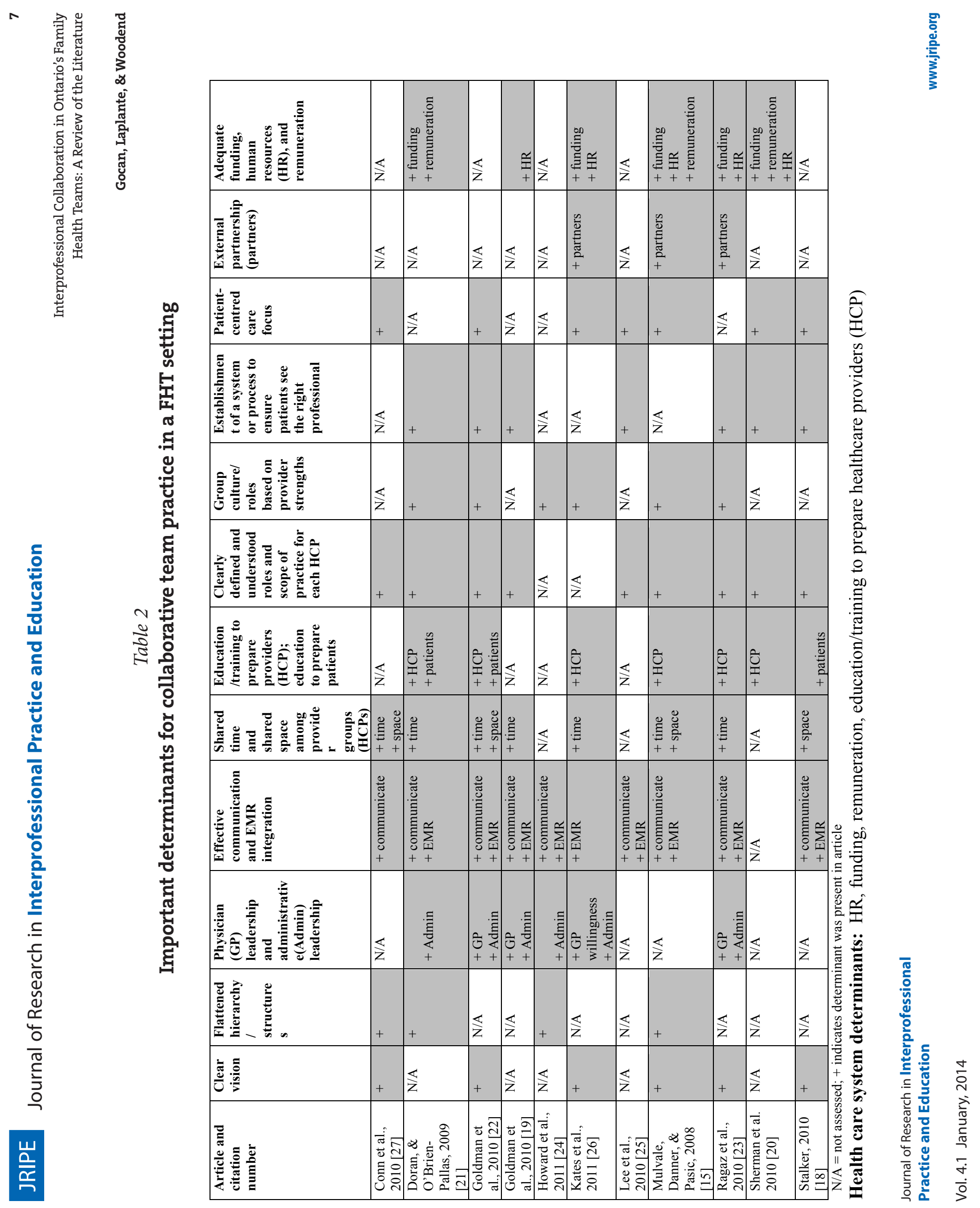


8

Interprofessional Collaboration in Ontario's Family Health Teams: A Review of the Literature

Gocan, Laplante, \& Woodend

Journal of Research in Interprofessional Practice and Education

Vol. 3.3

January, 2014 ence on FHTs, including broad healthcare system determinants, a FHTs local context, and team determinants within individual FHT organizations.

\section{Healthcare system determinants}

Factors that supported effective interprofessional collaboration within FHTs at the healthcare system-level included adequate funding, human resources, remuneration incentives, and degree of professional preparation for collaborative practice.

\section{Adequate funding, remuneration, and human resources}

Adequate funding for interdisciplinary health professionals (IHP) enabled FHT patients to access primary healthcare services that were not previously available, such as mental healthcare and chronic disease management programs [15,18]. Physician remuneration changes from fee-for-service to blended capitation were credited as an important facilitator of collaborative care and a promoter of patientcentred services [15]. Some teams, however, reported difficulty recruiting physicians or filling certain IHP positions, limiting the advancement of collaborative care activities and needed clinical programs [15,19]. Recruitment challenges were multifaceted, including geographic barriers with low representation of certain professional groups, as well as inadequate remuneration of select IHP groups with low salary benchmarks compared to those in the hospital sector [15,20,21]. In FHTs, funding for IHPs is based on the number of rostered patients under the care of a family physician; hence, family physician shortages also limited funding opportunities for IHP team members, making comprehensive care more difficult to provide [20].

\section{Degree of professional preparation for collaborative practice}

Six studies reported a need for enhanced professional preparation for collaborative practice $[15,20-23,24]$. Doran and O'Brien-Pallas described limitations in this area, reporting that only half of the professionals in their study received training for collaboration prior to FHT employment [21]. Moreover, they also noted that although $62.5 \%$ of the participating FHTs provided collaboration training for staff after joining the FHT, only one of the FHT organizations provided training to all team members, limiting the reach of this intervention [21]. Interprofessional educational forums were regarded as a means of increasing collaboration, enhancing understanding of professional roles, and supporting a team approach to care in day-today practice $[15,20-23,24]$.

\section{Local context determinants}

Characteristics specific to a FHT's local context included the degree of electronic medical record (EMR) integration and the formation of community alliances or program facilitation partnerships.

\section{EMR integration}

A highly functional EMR system that facilitated sharing and management of patient clinical data, decision making support, and order entry was deemed impor- 
9

Interprofessional Collaboration in Ontario's Family Health Teams: A Review of the Literature

Gocan, Laplante, \& Woodend

Journal of Research in Interprofessional Practice and Education

Vol. 3.3

January, 2014 tant for interprofessional collaboration $[19,22,25]$. EMRs were identified as a critical tool for consistency in clinical care, continuity, and communication, in addition to data management for quality improvement, program planning, and evaluation $[15,22,23,26]$. Teams described challenges in implementing EMR systems without dedicated human resources and identified a need to allocate EMR system management into the role of a staff member with technical expertise [23]. In some instances, challenges in updating EMRs limited the advancement of interprofessional team protocols and practices [19].

\section{Community alliances or program facilitation partnerships}

Three studies described benefits to collaborative team practice that resulted from inter-organizational and/or external partnerships and facilitation $[15,19,24]$. To enhance local healthcare delivery, FHTs have been encouraged to collaborate with community partners [17]. FHT groups recognized they were interdependent with community partners in order to meet the broad spectrum of patient needs. They discovered that co-ordinated partnerships were necessary for comprehensive mental healthcare and for the management of complex chronic health needs $[15,19,24]$. External project managers and facilitators were credited with providing assistance to teams in tool development, system planning, and project advancement, as FHT members found it difficult to dedicate time toward these tasks [19].

\section{Team determinants}

The final group of factors pertained to team determinants within individual FHT organizations. These included clarity of vision, hierarchical structure, use of a patient-centred approach to care, group culture, effective leadership, communication, shared time, and shared space. Other factors included adequate systems and processes to ensure that the right patient is seen by the right professional, clearly defined and understood roles and scope of practice, and patient education regarding each professional's role in the collaborative care process.

\section{Clarity of vision}

The FHT vision was viewed as an active process and philosophy of care in which team efforts were co-ordinated toward a larger goal [15]. A clear team vision defined team culture and set the foundation for collaborative practice. In and of itself, however, a vision inclusive of team practice did not translate directly into effective team functioning for FHTs [27]. Professional silos, spatial separation, and hierarchical structures precluded the establishment of a shared vision [27]. In order to translate a vision from words into actions, teams needed guidance and explicit support from administrative and clinical leaders, as well as dedication of financial and human resources [19].

\section{Group culture, flattened hierarchy, and effective leadership}

Effective leadership as well as mutual trust and respect within the team culture are essential in the team environment $[23,25]$. FHT leaders described strategies to 
10

Interprofessional Collaboration in Ontario's Family Health Teams: A Review of the Literature

Gocan, Laplante, \& Woodend

Journal of Research in Interprofessional Practice and Education

Vol. 3.3

January, 2014 develop the desired group culture, including targeted hiring based on key attributes such as flexibility, openness, leadership qualities, and degree of initiative [22,23]. Effective human resource policies were also identified; these policies fostered a collaborative team structure, clear guidelines surrounding expectations for practice, enhanced conflict resolution, and reduced professional hierarchies and disputes $[22,23]$. Effective administrative leadership unified team differences, enhanced problem solving, and provided support for innovative clinical practices [24,25].

Teams with a hierarchical structure reported negative effects from this work structure on teamwork [25]. However, shared power and accountability within group culture were challenging to operationalize [22]. Visible physician leadership was important to endorse team collaboration and provide role modelling for physician colleagues who were slower to embrace shared responsibility within the team, given the shift in expectations from solo-practitioner, independent practices [19,22-24]. A change in general discourse was suggested as a strategy to conceptualize the team differently; non-physician and physician staff were often separated in the language used by the team, serving as a barrier that restricted egalitarian advancement [27]. In addition, comments from patients or physicians that described staff as belonging to a physician, such as "my nurse," negated collaboration and reinforced a vertical hierarchy [21,27]. Current Canadian medico-legal responsibilities, wherein physicians shoulder primary accountability, were also seen as a barrier to shared responsibility and flattened hierarchy structures [27].

\section{Clearly defined and understood roles and scope of practice}

Healthcare professionals working at FHTs noted substantial changes, including uncertainty in their roles and responsibilities [22]. IHPs reported that working to their full scope of practice was a rewarding component of working in a FHT setting. However, many IHPs described the challenges and frustration of defining unique contributions and educating clinical and administrative colleagues about their roles $[21,23]$. In order to optimize team effectiveness, a careful balance between role clarity and role flexibility was described in which overlapping scopes could be delineated based on patient needs, unique professional skills, and individual comfort level $[15,23,24]$. For physicians in particular, engagement and education around the role of IHPs were important to reduce team tensions and help physicians transition from a self-reliant style of practice to a shared-care approach [15,21-23].

\section{A patient-centred approach to care and patient education regarding their role}

Teams that focused on patient-centered approaches to care were able to more readily engage in collaboration and innovation through their focus on meeting patient needs rather than organizing care according to professional convenience [15,18,27]. Patients, however, experienced misunderstandings regarding the FHT model of practice, as they were used to receiving care almost exclusively from their physician $[21,22]$. Not unlike all members of the FHT staff, patients also had learning needs; education of patients regarding interprofessional practice assisted patients in adjust- 
11

Interprofessional Collaboration in Ontario's Family Health Teams: A Review of the Literature

Gocan, Laplante, \& Woodend

Journal of Research in Interprofessional Practice and Education

Vol. 3.3

January, 2014 ing their expectations of care and facilitated patient participation in the team-based model of practice $[18,22]$.

\section{Systems and processes to ensure the right patient is seen by the right professional}

It was noted that the key to patient access and FHT efficiency was ensuring patients are seen by the right professional for their care [22]. Structured referral processes, triage systems, and interprofessional dialogue were used to assist in the navigation of care and to enhance the efficiency and effectiveness of team functioning [21$23,26]$. These strategies were credited with reducing wait times and streamlining care as well as expediting services for those at highest risk $[22,23,26]$. These systems were evidence-based and tailored to match team resources and population needs $[19,26]$. Interprofessional representation in the development of systems enhanced understanding of diverse professional roles and the establishment of practical and relevant team protocols [19]. Buy-in from key stakeholders and FHT leaders was essential during protocol planning and implementation to support changes from the status quo [19].

\section{Communication strategies, shared time and space}

Communication was an essential component for developing group culture and creating a shared sense of accomplishment within the team [23]. Multiple communication strategies were reported to keep administrative and healthcare team members informed and engaged. This included emails, EMR messaging, team meetings, interprofessional committees, case conferences, brief daily "team huddles," education rounds, team retreats, and hallway conversations [21-23,27]. Challenges in engaging physicians were reported in three studies, with physicians remaining peripheral to team meetings and unit events $[19,21,27]$. Office locations that were physically divided within a FHT organization (owing to poor layout of space or FHTs that were distributed across multiple sites) hindered opportunities for interprofessional collaboration and practice changes $[19,22]$. FHT staff highlighted the importance of shared time and space in fostering communication and collaborative care [15,27].

\section{Outcomes of a FHT approach to care}

Eight studies included evaluation data pertaining to patient, provider, and healthcare system outcomes (Table 3). These were primarily qualitative in nature.

\section{Enhanced access to primary care and extended health services}

Enhanced patient access to primary care services was described in all eight studies reporting outcomes. For patients, this was identified as one of the most significant benefits of participating in a FHT setting [18]. Availability of after-hours clinical services, reduced wait times, and IHP services were seen as key drivers to enhancement of patient access [18]. In addition to improved access to basic primary care service, participants reported enhanced access to extended health services and more holistic care compared to what was previously available $[19,21,22,24]$. In some 
12

Interprofessional Collaboration in Ontario's Family Health Teams: A Review of the Literature

Gocan, Laplante, \& Woodend
Journal of Research in Interprofessional Practice and Education

Vol. 3.3

January, 2014 cases, preventive health care, chronic disease management, and mental healthcare services were perceived as "add-on" components of care, rather than core services, despite their central role in primary care [20]. Physicians noted that the diversity of professional expertise available in a FHT practice allowed them to address patient concerns they did not previously have the resources or skills to attend to, thus changing their health management approaches [18].

\section{Improved co-ordination, collaboration, and patient centredness}

Teams described improvements in their ability to provide comprehensive, co-ordinated, patient-centred services [15,22,24,26-27]. They described efficiencies based on patient needs rather than the needs of the professional. For example, multiple provider visits were co-ordinated on the same date for patient convenience and respect for patient time and resource constraints [27]. For patients, co-ordination of care through their primary care provider resulted in "one stop" services, improved patient choice, motivation for self-management participation, and enhanced comfort and ease with which they received health services $[18,20,24]$.

\section{Clinical outcomes}

There was a consensus across research studies that interprofessional collaboration among FHT practitioners generated improved clinical outcomes for patient care in areas such as health promotion, disease prevention, chronic disease management, and mental health services $[15,18,21,24]$.

\section{Patient satisfaction}

Enhanced patient satisfaction with care was described in five (out of eight) studies reporting outcomes [18,22,24,26-27]. Enhancements to patient access, comprehensive care, co-ordination of care, patient centred care, adequate time during appointments, and opportunities for shared decision making were credited for these improvements [18,22].

\section{Provider outcomes}

FHT professionals involved in interprofessional collaboration reported greater understanding of professional roles [22] and improvements in their professional skills and capabilities [24,26]. Moreover, physicians reported a greater sense of satisfaction within a FHT model of practice [18]. They indicated that working within a team provided increased collegial support, afforded them more time to spend with patients, and enhanced access to the resources needed to provide quality patient care [18].

\section{Healthcare system outcomes}

\section{Increased healthcare access and efficiency}

Providers reported improvements in the standardization of screening practices and accessibility of services [19]. Improved triage systems and referral processes were 


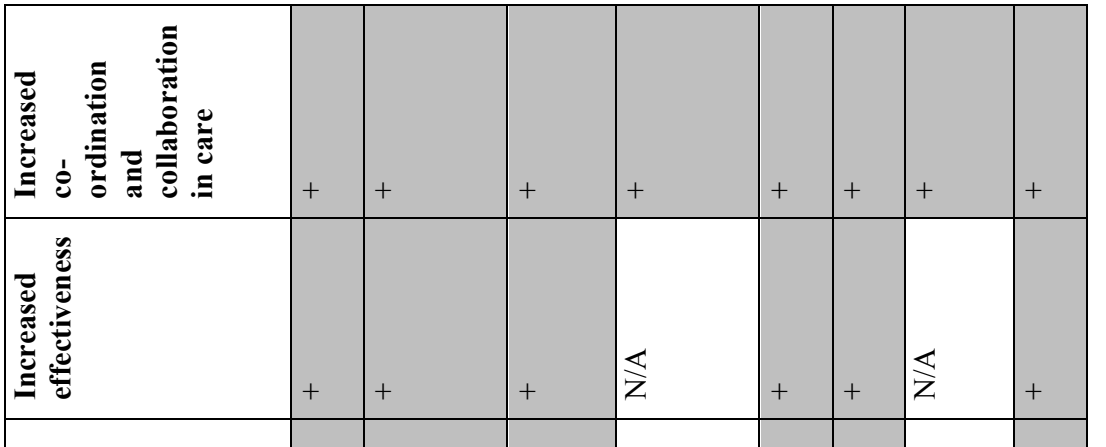

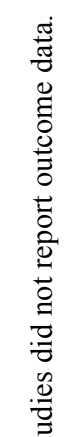
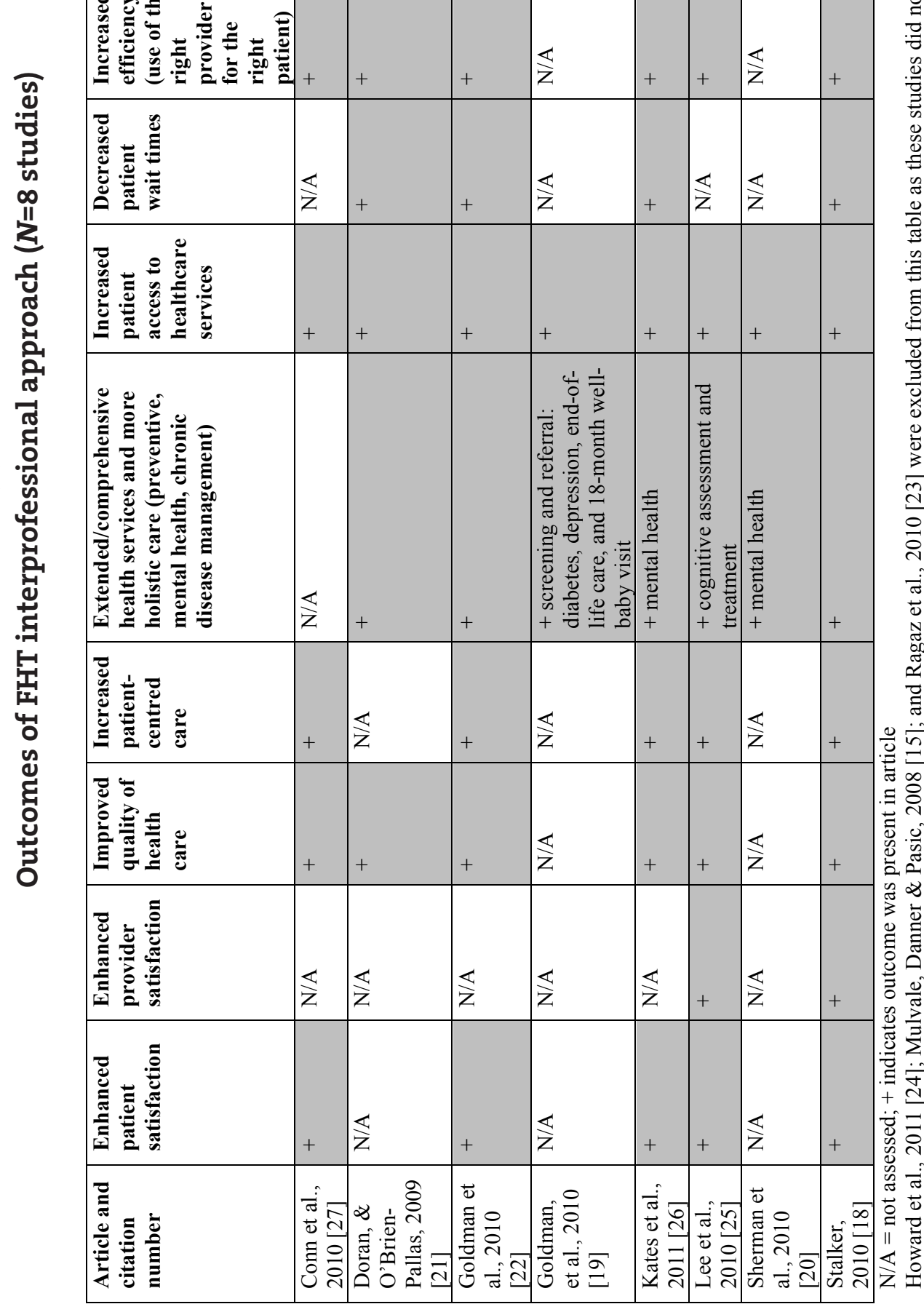
14

Interprofessional Collaboration in Ontario's Family Health Teams: A Review of the Literature

Gocan, Laplante, $\&$ Woodend
Journal of Research in Interprofessional Practice and Education

Vol. 3.3

January, 2014 described which helped to ensure the right patients were seen by the right provider for the right health concern $[19,25]$. System navigation services also helped patients to access care more readily in their communities $[20,26]$.

\section{Increased effectiveness}

Staff described improvement for patients who had been at a standstill in their health progress and credited broad commitment to working as a team [27]. More effective use of health resources, enhanced community partnerships, and improvements in clinical outcomes were also reported [19,21,24,25]. In addition, emergency department use decreased [21].

\section{Changes in wait times}

Decreased wait times were identified by both patients and providers as an enhancement to care [18,24]. Patient wait times decreased for primary care appointments, diagnostic testing, and services such as mental health assessment $[18,24]$. In some cases, however, despite increased availability of social work or psychiatry services, for example, accessibility issues remained as a result of high referral volumes or eligibility restrictions $[18,20]$. Patients also noted that although appointments in general were readily available with a variety of health professionals within the team, they experienced a longer wait to see their own personal physician [18].

\section{Discussion}

This review and analysis of the literature was conducted to establish a deeper understanding of FHT team functioning in Ontario. There are a number of common challenges to interprofessional collaboration within FHTs despite their clear mandate for team-based practice and Ontario Ministry of Health guidelines for establishing collaborative care. Notwithstanding these challenges, there is some evidence that interprofessional collaboration is gradually taking shape within these team-based practices, and several positive outcomes have been identified through qualitative research in FHTs.

Patient and provider perceptions around the outcomes of collaborative care in a FHT setting indicate that interprofessional teams were able to provide enhanced access to care and extended healthcare services compared to what had previously been offered in a uniprofessional model of care. Both patients and providers experienced more time for care and enhanced quality of health services. Interprofessional collaboration also assisted providers with shifting their approach to healthcare, addressing mental health or chronic disease concerns they previously avoided given their lack of professional resources or expertise. Providers described changes to health management approaches that were directly related to having access to skilled IHPs who could assist patients to effectively cope with diverse aspects of their health condition(s).

This literature review reinforces what has previously been found in research looking at interprofessional collaboration and team functioning within other healthcare settings. For example, in keeping with prior studies and systematic 
15

Interprofessional Collaboration in Ontario's Family Health Teams: A Review of the Literature

Gocan, Laplante, \& Woodend

Journal of Research in Interprofessional Practice and Education

Vol. 3.3

January, 2014 reviews, shared time and clinical spaces were seen as essential building blocks for team cohesion and collaborative practice [14,28]. In addition, interpersonal aspects of care such as group culture, leadership, and EMR functionality were found to be significantly more predictive of team climate than the organizational composition of the teams (such as number of health professionals on staff, practice size, number of sites, governance structure) [25]. This was also noted by Sicotte et al. in their analysis of CCHCs in Quebec, where contextual factors were found to have a limited impact on interprofessional collaboration compared to the significant effects of internal work group dynamics [12].

A clear team mission, established goals, and operational objectives have also been identified in previous research as important to the foundation of effective teamwork $[29,30]$. The importance of a clearly defined vision inclusive of the team approach to care was identified in more than half of the research articles included in the current synthesis. Together with clear team objectives, specific, measurable operational goals were seen as an important determinant of team success [23]. It was acknowledged that in the early development of FHTs, evaluation systems may assume a low priority; however, their use provides teams with important benchmark data and impact assessment outcomes, assisting teams with resource allocation and program planning [23]. In previous research, formal assessment or evaluation of quality of care outcomes and program achievements were found to influence the intensity of collaboration [12].

The importance of strong leadership was also a notable determinant for effective FHT functioning. This leadership provided the groundwork for mutual trust and respect within team culture, and fostered a supportive work environment [23,26]. Leadership from family physicians was identified as an important factor in achieving shared responsibility, accountability, and reducing hierarchical structures $[19,22,23,24]$. Mutual interdependency, partnership, and symmetry in power have also been recognized as key concepts in interprofessional collaboration $[14,30]$. The disciplinary isolation in which health providers are educated is a known barrier to collaboration [14]. The important role of training, role modelling, effective leadership, continuing education, and facilitation in the refinement of team culture and collaborative practices were identified in this review [21-23].

This review builds on the knowledge that health teams benefit from training in collaborative care [14]. Training among patients is also needed to enhance their ability to take an active role in collaboration. Several studies indicated that patients needed to adjust to the group structure of FHTs and benefited from education regarding IHP roles [21,22]. Miscommunication and inadequate knowledge regarding professional roles resulted in patient resistance to care from IHPs, and underutilization of provider resources [21]. A lack of patient understanding around professional roles could also limit full participation by team members and impede improvements in patient outcomes that could be achieved through shared disciplinary expertise. Visible team care has been described as care in which the roles of each health provider are known and understood by the patient [7]. When invisible team care occurs, the roles and identities of IHPs are not clear to the patient and 
16

Interprofessional Collaboration in Ontario's Family Health Teams: A Review of the Literature

Gocan, Laplante, \& Woodend

Journal of Research in Interprofessional Practice and Education

Vol. 3.3

January, 2014 these healthcare providers are referred to as "not my doctor" [7]. Knowledge gaps exist in the examination of patient perspectives and patient readiness for collaborative roles within interprofessional models of care.

The importance of collaborative processes, including co-ordination and communication mechanisms, has previously been identified as a core concept within interprofessional collaboration $[14,30]$. This includes the establishment of processes or systems to assist patients in accessing the right provider for the right health concern. EMR integration and partnership/facilitation support are less studied elements that were identified for their importance in establishing enhanced collaborative processes of care. Dedicated human resources, funding, and technical expertise were essential to EMR system integration and the advancement of interprofessional team protocols and practices $[19,23]$. Significant benefits resulted from co-ordinated partnerships and facilitation support for tool development, system planning, and team progress in the delivery of health promotion, preventive programs, and chronic disease prevention and management $[15,19,24]$.

Strengths of this literature review include the identification of research studies that collectively involved 87 FHT organizations, 694 FHT staff members, and 80 patients/family members. FHT participants were represented by a variety of interprofessional team members and diverse FHT settings. Key determinants of interprofessional collaboration identified through these research studies were reinforced by what has been previously published in systematic reviews.

Limitations of this study included low levels of quantitative research evidence and an underrepresentation of the patient's perspective as a key participant in interprofessional collaboration.

\section{Implications for practice, education, research, and public policy}

FHTs are proliferating in Ontario; as such, considerable focus and attention should be made to expedite and optimize interprofessional team functioning, thereby facilitating the achievement of improved accessibility, effectiveness, and quality of primary care health services across the province.

Healthcare providers and administrative staff in FHTs are not alone in their need for preparation to collaborate in the advancement of high-quality patient care. While there is a definite need for professional education among health providers and administrative staff, it is also important to recognize that patient factors influence the quality of interprofessional collaboration. As a key participant in shared decision-making and team-based care, patients need education and practical support to assume their central role within interprofessional teams. Accessing the right provider for the right health concern requires patients to be aware of the various scopes of practice and skills provided by a variety of healthcare practitioners.

D'Amour et al. have described gaps within interprofessional frameworks of care that have poorly conceptualized the role of patients in the collaborative process [30]. Patients and professionals alike need support to optimize their use of interprofessional models of practice. Further research is therefore needed to examine strategies that integrate patients more fully in the collaborative process. This should 
17

Interprofessional Collaboration in Ontario's Family Health Teams: A Review of the Literature

Gocan, Laplante, \& Woodend
Journal of Research in Interprofessional Practice and Education

Vol. 3.3

January, 2014 incorporate the perspective of patients to further understand their needs and enhance interprofessional collaboration in a fashion that endorses the patients' lead role in their healthcare.

Evaluation of concrete operational measures must also become a higher priority amongst FHT leaders, clinicians, and funding agencies. There is a paucity of quantitative data regarding FHT outcomes to date. Internal assessment of quality of care and program accomplishments can enhance the intensity of interprofessional collaboration [12]. Integrated supports and prioritization for quality improvement planning cycles and evaluation research are greatly needed in Ontario FHTs. The integration of EMRs in all FHTs was in part to facilitate evaluation of local population needs and program effectiveness [31]. Internal evaluation and feedback may in and of itself provide a means to intensify collaboration through the identification of successful programs, effective team partnerships, and reinforcement of collective actions that result in positive outcomes. Publication of evaluation findings is important for the replication of successful strategies and sharing of programs that demonstrate the greatest patient benefit across Ontario FHTs.

It is not enough for policy makers to establish interprofessional primary care practices. Careful planning is needed in the development of shared work space and supported EMR systems that facilitate easy communication and interprofessional program development. In addition to adequate funding, a need was identified for a funding model that remunerates all members of the interprofessional team in a way that supports interprofessional care and collaboration.

Policy makers need to provide leadership in the prioritization and standardization of preventive care, mental health services, and chronic disease management as core elements of primary healthcare rather than allowing these essential services to be regarded as optional or "add-on" components of care. For healthcare teams, evidence-based educational interprofessional programs, community partnerships, and facilitation support may prove to be useful strategies to more fully develop provider skills and competence in the delivery of comprehensive primary health services, providing benefits and positive outcomes for both patients and providers alike.

\section{Abbreviations}

CHCCs - Community Health Care Centres

EMR(s) - Electronic Medical Record(s)

FHT(s) - Family Health Team(s)

GP - General Practitioner/Physician

$\mathrm{HCP}(\mathrm{s})$ - Health Care Provider(s)

HR - Human Resources

IHP(s) - Interdisciplinary Health Professional(s)

\section{References}

1. Health Canada. (2006). 2003 First Ministers Health Accord. URL: http://www.hc-sc.gc.ca/hcssss/delivery-prestation/fptcollab/2003accord/primary-primaire-eng.php [May 13, 2012].

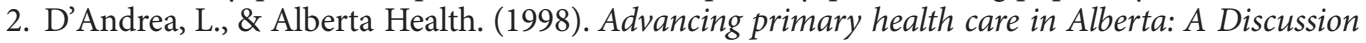

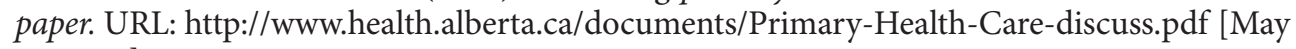
13, 2012]. 
18

Interprofessional Collaboration in Ontario's Family Health Teams: A Review of the Literature

Gocan, Laplante, \& Woodend

Journal of Research in Interprofessional Practice and Education

Vol. 3.3

January, 2014
3. Nolte, J., \& Tremblay, M. (2005). Enhancing interdisciplinary collaboration in primary health care in Canada. Ottawa, ON: Enhancing Interdisciplinary Collaboration in Primary Health Care Initiative.

4. Canadian Nurses Association. (2005). Primary health care: A summary of the issues. Ottawa, ON: Canadian Nurses Association.

5. Way, D., Jones, L., \& Busing, N. (2000). Implementation strategies: "Collaboration in primary care -Family doctors \& nurse practitioners delivering shared care": Discussion paper written for the Ontario College of Family Physicians. Ottawa, ON: Ontario College of Family Physicians.

6. Barrett, J., Curran, V., Glynn, L., \& Godwin, M. (2007). CHSRF synthesis: Interprofessional collaboration and quality primary healthcare. Ottawa, ON: Canadian Health Services Research Foundation.

7. Safran, D.G. (2003). Defining the future of primary care: What can we learn from patients? Annals of Internal Medicine 138, 248-255.

8. Curran, V. (2007). Collaborative care: Synthesis series on sharing insights. Ottawa, ON: Health Canada.

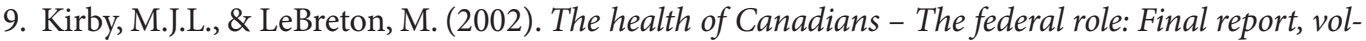

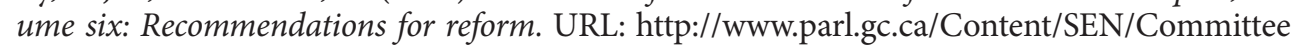
/372/SOCI/rep/repoct02vol6-e.htmi:[May 13, 2012].

10. Mable, A.L., \& Marriott, M. (2002). Sharing the learning: The health transition fund: Synthesis series: Primary health care. Ottawa, ON: Minister of Public Works and Government Services Canada.

11. Bailey, P., Jones, L., \& Way, D. (2006). Family physician/nurse practitioner: Stories of collaboration. Journal of Advanced Nursing 53, 381-391.

12. Sicotte, C., D’Amour, D., \& Moreault, M. (2002). Interdisciplinary collaboration within Quebec community health care centres. Social Science \& Medicine 55, 991-1003.

13. Légaré, F., Stacey, D., Gagnon, S., Dunn, S., Pluye, P., Frosch, D., Kryworuchko, J., Elwyn, G., Gagnon, M-P., \& Graham, I.D. (2010). Validating a conceptual model for an inter-professional approach to shared decision making: A mixed methods study. Journal of Evaluation in Clinical Practice, 1-11.

14. San Martin-Rodriguez, L., Beaulieu, M., D’Amour, D., \& Ferrada-Videla, M. (2005). The determinants of successful collaboration: A review of theoretical and empirical studies. Journal of Interprofessional Care 19, 132-147.

15. Mulvale, G., Danner, U., \& Pasic, D. (2008). Advancing community-based collaborative mental health care through interdisciplinary family health teams in Ontario. Canadian Journal of Community Mental Health 27, 55-73.

16. Ministry of Health and Long-Term Care. (2011). FHT to print: A newsletter for Ontario's family health teams: Summer/Fall 2011. Toronto, ON: Ministry of Health and Long-Term Care.

17. Ministry of Health and Long-Term Care. (2009). Roadmap to implementing a Family Health Team. Toronto, ON: Queen's Printer for Ontario.

18. Stalker, C.A. (2010). How have physicians and patients at New Vision family health team experienced the shift to a family health team model? A pilot study: Final report. Waterloo, ON: Lyle S. Hallman Faculty of Social Work.

19. Goldman, J., Meuser, J., Lawrie, L., Rogers, J., \& Reeves, S. (2010). Interprofessional primary care protocols: A strategy to promote an evidence-based approach to teamwork and the delivery of care. Journal of Interprofessional Care 24, 653-665.

20. Sherman, J.E., Pong, R.W., Swenson, J.R., Delmege, M.G., Rudnick, A., Cooke, R.W., Ravitz, P., \& Montgomery, P. (2010). Mental health services in smaller northern Ontario communities: A survey of Family Health Teams. Sudbury, ON: Centre for Rural and Northern Health Research in collaboration with The Ontario Psychiatric Outreach Program.

21. Doran, D., \& O’Brien-Pallas, L. (2009). An evaluation of communication practices in Ontario family health teams (FHT): Final report. Toronto, ON: Nursing Health Services Research Unit.

22. Goldman, J., Meuser, J., Rogers, J., Lawrie, L., \& Reeves, S. (2010). Interprofessional collaboration in family health teams: An Ontario-based study. Canadian Family Physician 56, e368-e374.

23. Ragaz, N., Berk, A., Ford, D., \& Morgan, M. (2010). Strategies for family health team leadership: Lessons learned by successful teams. Healthcare Quarterly 13, 39-43.

24. Kates, N., McPherson-Doe, C., \& George, L. (2011). Integrating mental health services within primary care settings: The Hamilton family health team. Journal of Ambulatory Care Management 34, 174-182. 


\section{JRIPE

19

Interprofessional Collaboration in Ontario's Family Health Teams: A Review of the Literature

Gocan, Laplante, \& Woodend
25. Howard, M., Brazil, K., Akhtar-Danesh, N., \& Agarwal, G. (2011). Self-reported teamwork in family health team practices in Ontario: Organizational and cultural predictors of team climate. Canadian Family Physician 57, e185-191.

26. Lee, L., Hillier, L.M., Stolee, P., Heckman, G., Gagnon, M. et al. (2010). Enhancing dementia care: A primary care-based memory clinic. Journal of the American Geriatrics Society 58, 2197-2204.

27. Conn, L.G., Oandasan, I.F., Creede, C., \& Jakubovicz, D. (2010). Creating sustainable change in the interprofessional academic primary care setting: An appreciative inquiry approach. Journal of Research in Interprofessional Practice and Education 1(3), 284-300.

28. Oandasan, I.F., Conn, L.G., Lingard, L., Karim, A., Jakubovicz, D., Whitehead, C., Miller, K.L., Kennie, N., \& Reeves, S. (2009). The impact of space and time on interprofessional teamwork in Canadian primary health care settings: Implications for health care reform. Primary Health Care Research \& Development 10, 151-162.

29. Grumbach, K., \& Bodenheimer, T. (2004). Innovations in primary care. Can health care teams improve primary care practice? Journal of the American Medical Association 291, 1246-1251.

30. D’Amour, D., Ferrada-Videla, M., Rodriguez, L.S.M., \& Beaulieu, M. (2005). The conceptual basis for interprofessional collaboration: Core concepts and theoretical frameworks. Journal of Interprofessional Care 19, 116-131.

31. Ministry of Health and Long-Term Care. (2005). Guide to information technology. Toronto, ON: Ministry of Health and Long-Term Care. 